\title{
Perception of acceleration with short presentation times: Can acceleration be used in interception?
}

\author{
ANNE-MARIE BROUWER, ELI BRENNER, and JEROEN B.J. SMEETS \\ Erasmus University Rotterdam, Rotterdam, The Netherlands
}

\begin{abstract}
To investigate whether visual judgments of acceleration could be used for intercepting moving targets, we determined how well subjects can detect acceleration when the presentation time is short. In a differential judgment task, two dots were presented successively. One dot accelerated and the other decelerated. Subjects had to indicate which of the two accelerated. In an absolute judgment task, subjects had to adjust the motion of a dot so that it appeared to move at a constant velocity. The results for the two tasks were similar. For most subjects, we could determine a detection threshold even when the presentation time was only $300 \mathrm{msec}$. However, an analysis of these thresholds suggests that subjects did not detect the acceleration itself but that they detected that a target had accelerated on the basis of the change in velocity between the beginning and the end of the presentation. A change of about $25 \%$ was needed to detect acceleration with reasonable confidence. Perhaps the simplest use of acceleration for interception consists of distinguishing between acceleration and deceleration of the optic projection of an approaching ball to determine whether one has to run backward or forward to catch it. We examined the results of a real ball-catching task (Oudejans, Michaels, \& Bakker, 1997) and found that subjects reacted before acceleration could have been detected. We conclude that acceleration is not used in this simple manner to intercept moving targets.
\end{abstract}

In the present study, we investigate the possibility of using information about targets' angular acceleration ${ }^{1}$ when one is intercepting moving targets. For successful interception, it is necessary to take the changing position of the moving target into account. Information about the target's acceleration could therefore be helpful. When the target moves in a frontal plane (as was the case in the interception studies of Bairstow, 1987; Brouwer, Brenner, \& Smeets, 2000; van Donkelaar, Lee, \& Gellman, 1992), any acceleration of the target will result in an (approximately) equivalent optic angular acceleration. Both Rosenbaum (1975) and Port, Lee, Dassonville, and Georgopoulos(1997) examined whether subjects took acceleration into account when predicting the time at which a target, moving in a frontal plane, reached a certain point in space. Rosenbaum presented accelerating targets moving from left to right, or vice versa. The targets disappeared behind a mask, and the task was to indicate when they passed a mark on the mask. Port et al. let their subjects intercept accelerating targets on a computer screen with a cursor controlled by a computer mouse. They were to intercept the targets within a prescribed area on the screen. Whereas Rosenbaum concluded that subjects used acceleration, Port et al. concluded

We thank Raoul Oudejans and Claire Michaels for providing us with the data from their experiment. A.-M.B. was supported by the Netherlands Organization for Scientific Research (NWO Grant 575-23 015). Correspondence should be addressed to A.-M. Brouwer, Max-PlanckInstitut für Biologische Kybernetik, Spemannstraße 38, 7206 Tübingen, Germany (e-mail: anne-marie.brouwer@tuebingen.mpg.de). that they did not. Perhaps subjects cannot use acceleration in a real interception task such as in Port et al., whereas they can use it in a more perceptual or cognitive task such as that of Rosenbaum. Another possible reason for the difference is that the accelerations of the stimuli used by Port et al. may not have been large enough. They were well above threshold levels found in other studies, but the durations of the stimuli in those studies were long, which, as we will argue later, can make a difference.

The relation between the acceleration of the target and the optic angular acceleration is not as simple when objects approach an observer, as is the case in catching fly balls. Nevertheless, even in this task, detecting angular acceleration can be helpful, though not for predicting when the target will be at a certain position. The sign of the angular acceleration of the vertical motion of the ball's projection indicates whether the ball will land ahead of or behind the observer (Chapman, 1968). Thus, subjects could in principle use angular acceleration to determine whether they should run forward or backward. Some research suggests that this strategy is used (Babler \& Dannemiller, 1993; Michaels \& Oudejans, 1992), and other research claims that it is not (McBeath, Shaffer, \& Kaiser, 1995; Todd, 1981). We will elaborate on this issue in the Discussion section.

It is difficult to determine whether humans perceive angular acceleration well enough to be able to use it in interception. Previous studies have reported very different detection thresholds for acceleration (Babler \& Dannemiller, 1993; Calderone \& Kaiser, 1989; Gottsdanker, Frick, \& Lockard, 1961; Schmerler, 1976). Moreover, the total 
change of velocity during a presentation predicts the detection threshold better than does the rate of change in velocity (Gottsdanker et al., 1961; Schmerler, 1976). Thus subjects appear to be unable to perceive the rate of change in velocity (the acceleration) and detect that the velocity has changed during the presentation instead. In the following, we will summarize some of these findings.

Gottsdanker et al. (1961) asked their subjects to discriminate between an accelerating dot and a dot moving at a constant velocity. The dots were presented successively, and there were separate sessions for positive and negative acceleration. Gottsdanker et al. reported a wide range of $75 \%$ correct thresholds. The thresholds varied between $26 \%$ and $157 \%$ change in velocity (hereafter defined as the difference between the final and initial velocity divided by the average velocity). The highest thresholds were found with the shortest presentation time, which was $450 \mathrm{msec}$. Schmerler's (1976) subjects categorized stimuli as having positive, negative, or no acceleration. There were separate sessions in which positively accelerating stimuli were presented together with nonaccelerating stimuli, and ones in which negatively accelerating stimuli were presented with nonaccelerating stimuli. The threshold was defined as the ratio between initial and final velocity that was required for $50 \%$ of the responses to be "positive acceleration" in the former sessions, and $50 \%$ of the responses to be "negative acceleration" in the latter sessions. The average threshold was a ratio of about 2.7 (which corresponds to a $92 \%$ change in velocity).Calderone and Kaiser (1989) used a task whereby subjects had to indicate whether or not a small square accelerated positively. In a separate condition, they had to indicate whether or not it decelerated. Calderone and Kaiser found a 75\% correct threshold of about $60 \%$ change in velocity. Subjects of Babler and Dannemiller (1993) indicated whether a dot accelerated positively or negatively. The $75 \%$ correct threshold was approximately $20 \%$ change in velocity.

Beside the fact that there is no agreement about threshold values between these studies, there is another reason why it is difficult to use these thresholds to determine whether acceleration is perceived well enough to be used in interception. Most stimuli in these studies were presented for $1 \mathrm{sec}$ or longer. In interception tasks, it is often necessary to react quickly. Thus, in order to be useful, acceleration has to be detected quickly. In the present study, we determined acceleration thresholds for presentation times shorter than a second.

We used two methods to determine acceleration thresholds: a differential judgment method and an absolute judgment method. In the differential judgment method, we successively presented the subjects with a positively and a negatively accelerating dot. The subjects had to indicate whether the first or the second moving dot accelerated positively. We used a staircase procedure to find a $75 \%$ correct threshold. In the absolute judgment method, the subjects had to adjust the acceleration of a moving dot so that the dot moved at a constant speed. This method has the advantage of revealing possible biases in what is perceived as a constant speed, which will affect interception behavior if acceleration is used.

\section{METHOD}

\section{Materials and Design}

The stimuli were presented at $120 \mathrm{~Hz}$ on a computer monitor (Silicon Graphics GTX-210 computer and Taxan Ergovision 2150 TC095 monitor). The spatial resolution was $815 \times 611$ pixels $(39.2 \times$ $29.3 \mathrm{~cm}$ ) and was enhanced with anti-aliasing techniques. The viewing distance was $50 \mathrm{~cm}$ (thus $1 \mathrm{~cm}$ on the screen corresponds to about $1.1^{\circ}$ of visual angle). A chin- and forehead rest was used to keep the viewing distance constant. The experiment took place in a normally illuminated room.

Subjects viewed a gray background with two $4-\mathrm{cm}$ red squares. A 4-mm diameter black dot appeared from behind the left square, moved to the right, and disappeared behind the right square. The dot was presented for 300,600 , or $900 \mathrm{msec}$ and had a mean velocity of 3,13 , or $23 \mathrm{~cm} / \mathrm{sec}$. This resulted in nine conditions (three presentation times $*$ three average velocities). The distances traveled by the dot (i.e., the separation between the red squares) necessarily differed between the conditions. Since we kept the average velocity and the duration constant within a condition, a change in acceleration resulted in a change of both the initial and the final velocity. The conditions were blocked, and the block order was randomized across subjects.

We tried to create circumstances favorable for the perception of acceleration. The two squares and the edges of the monitor were clearly visible and served as landmarks. Such landmarks have been shown to facilitate the perception of motion (see, e.g. Smeets \& Brenner, 1994). Furthermore, the design was blocked, so that the average speed was known in advance. Pursuit eye movements may be important in the perception of acceleration, and a predictable average speed will probably improve the quality of pursuit. The thresholds we obtained would therefore presumably be lower than those obtained in more natural situations.

Differential judgment task. Every condition was tested within a separate block of 50 trials. Two dots were presented in each trial, with an interstimulus interval of $1,000 \mathrm{msec}$. One of the dots accelerated positively and the other negatively, at the same rate. The task of the subject was to indicate whether the first or the second stimulus accelerated positively by pressing the appropriate mouse button.

We used a staircase method to find the acceleration at which subjects responded correctly on $75 \%$ of the trials. On the first trial, the dots moved at the maximal possible acceleration (defined by a zero starting velocity of one dot and a zero ending velocity of the other). The step size decreased logarithmically with acceleration rate, so that after each correct choice the acceleration was $80 \%$ of its previous value. After an error, the acceleration was increased by three such steps (to $195 \%$ of its previous value).

Absolute judgment task. Every condition was represented by a block of 20 trials. In each trial, one dot was presented repeatedly with a 1,000-msec interval between presentations. The subjects had to adjust the dot's acceleration with a computer mouse until the dot appeared to move at a constant velocity. We told them that moving the mouse to the right would increase the dot's acceleration and moving the mouse to the left would decrease its acceleration. The subjects were allowed to take as long as they liked (and thus to see the stimulus as often as they liked) to make their settings. Once they were satisfied, they pressed a mouse button. This started the next trial. The initial acceleration was chosen at random from the range of possible accelerations for that condition (limited by starting and ending velocities of 0 ).

\section{Analysis}

Differential judgment task. For each subject and each condition, the average percentage correct per value of acceleration was plotted 
as a function of the log of acceleration. The threshold was determined by fitting a sigmoid through these points and taking the intersection with the $75 \%$ correct line. The points were weighted by the square root of the number of times they were presented. The sigmoid was defined as

$$
y=50+\frac{50}{1+e^{a-b x}},
$$

where $x$ is $\log$ acceleration and $y$ is the average percentage correct. The values of $a$ and $b$ were fitted. This sigmoid starts at $y=50$ (chance level) for no acceleration and tends toward $y=100$ (always correct) for large accelerations.

As already mentioned in the introduction, it has been proposed that acceleration itself is not perceived, but that it is detected by comparing the initial and final velocity. We therefore also converted the thresholds into percentages of change in velocity (the difference between the final and initial velocity, divided by the mean velocity; Babler \& Dannemiller, 1993; Calderone \& Kaiser, 1989). For this calculation, we considered the dot's velocity when it was half occluded by each of the squares as its initial or final velocity.

Absolute judgment task. The average setting will reveal any bias in the perception of acceleration. However, a correct average setting (zero) does not tell us that acceleration is perceived well; it will also be about zero if subjects set the acceleration values at random. We used the standard deviation of the settings per subject and condition as acceleration thresholds. These thresholds were also converted into percentages of change in velocity.

Statistical tests. We used repeated measures analyses of variance to examine the effects of presentation time and of average velocity on the thresholds expressed both as the logarithm of acceleration in $\mathrm{cm} / \mathrm{sec}^{2}$ and as the percentage of change in velocity. The biases of the settings were evaluated with one-sample $t$ tests. The input for these tests was the average setting for each subject and condition. We took $p<.05$ as the level of significance.

\section{Subjects}

Ten observers participated. Three of them were the authors. The other subjects were naive about the purpose of the experiment. Subjects could take a break between blocks.

We excluded the results of 3 (naive) subjects in both tasks from analysis because these subjects could not discriminate between the maximal accelerations in one or more conditions of the differential judgment task (operationalized as this maximal value's having been presented more than 10 times, which made it impossible to determine a threshold). Excluding the subjects poorest at perceiving accelerations contributes to finding low thresholds.

\section{RESULTS}

\section{Differential Judgment Task}

Figure 1A shows the raw staircases of a subject who cannot distinguish between positive and negative acceleration (s1) and a subject who can (s2) in the condition of $300-\mathrm{msec}$ presentation time and $3 \mathrm{~cm} / \mathrm{sec}$ average velocity. Even at the maximal acceleration of $20 \mathrm{~cm} / \mathrm{sec}^{2}$, subject $\mathrm{s} 1$ does not respond above chance. Thus, a threshold cannot be determined. This subject is one of the 3 who were excluded from further analysis. Figure 1B shows the sigmoid fit for Subject s2.

The mean acceleration thresholds (Figure 2A) clearly depend on presentation time $[F(2,12)=177.01, p<.01]$ and mean velocity $[F(2,12)=59.74, p<.01]$; the longer the dot is visible and the slower its mean velocity, the lower the threshold. In Figure 2B, the thresholds are ex- pressed as percentages of change in velocity. When described in this manner, the thresholds still depend on presentation time $[F(2,12)=9.57, p<.01]$, but less conspicuously than when they are described as accelerations. Thresholds expressed as percentages of change in veloc-
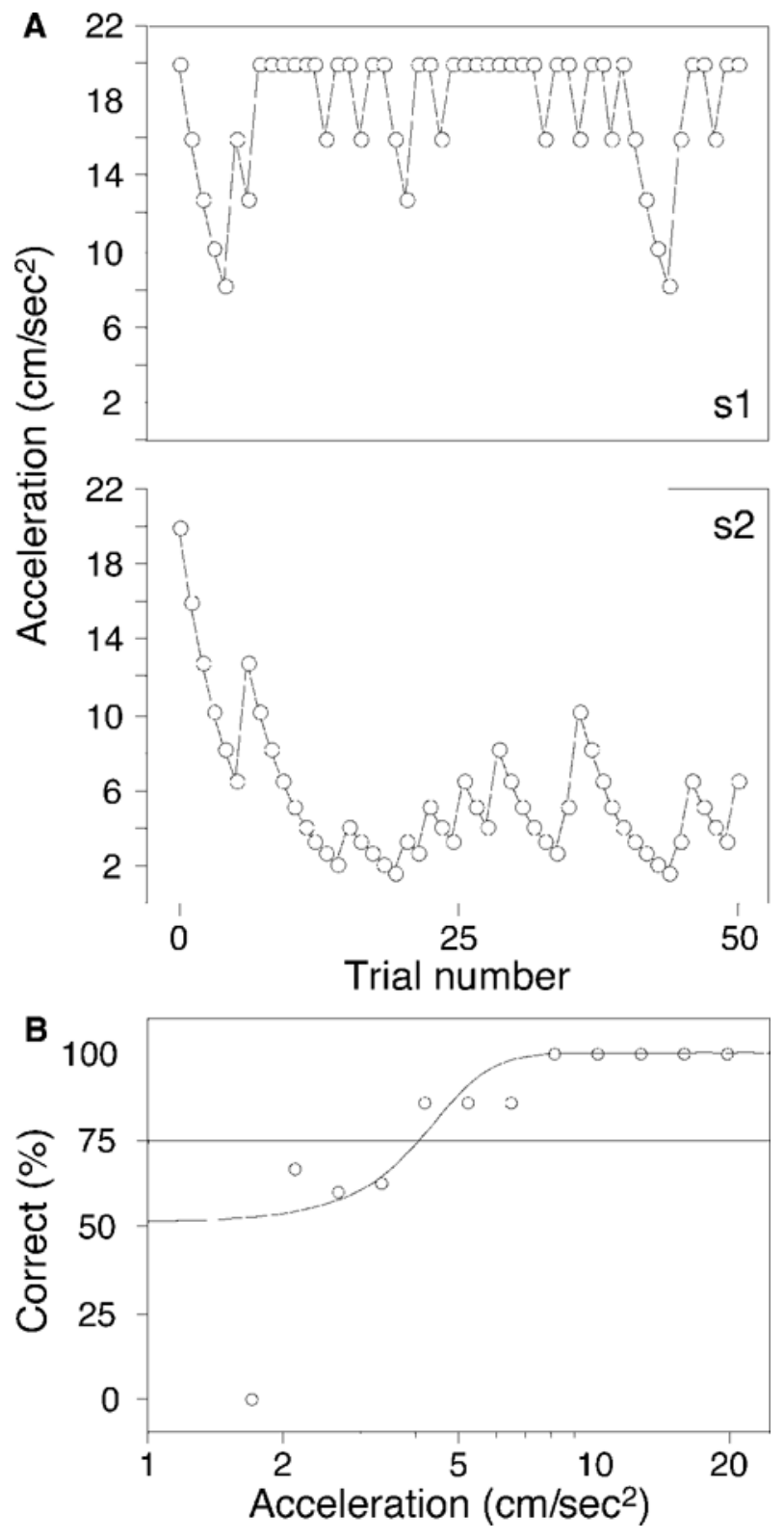

Figure 1. Examples of 2 subjects' staircases (A) and 1 subject's fitted sigmoid $(B)$ in the condition with $300-\mathrm{msec}$ presentation time and a mean velocity of $3 \mathrm{~cm} / \mathrm{sec}$. When the subject labels the correct dot as positively accelerating, the acceleration of the dots in the next trial is one step lower. When he or she makes a mistake, the acceleration increases three steps. Subject s1 is unable to distinguish between positive and negative acceleration even at the extremes. This subject was 1 of the 3 whom we excluded from further analysis. Subject $\mathrm{s} 2$ reaches a threshold value. The threshold is defined as the acceleration where the fitted sigmoid crosses the $75 \%$ correct line. 

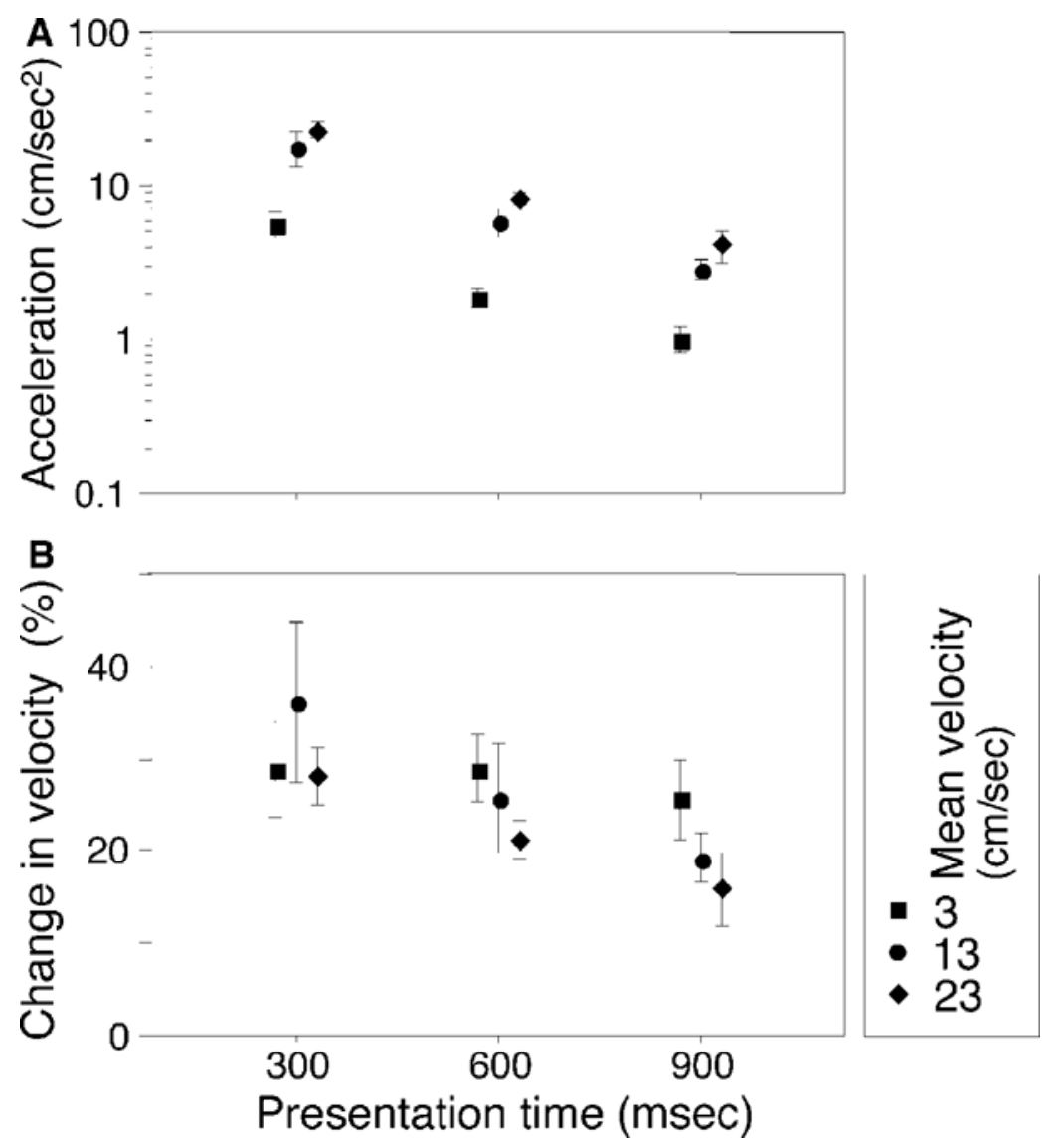

Figure 2. Results of the differential judgment task. Mean thresholds of 7 subjects with between-subjects standard errors for each condition. The thresholds are expressed both as rate of change in velocity (A) and as percentage of change in velocity (B). In the former unit, the thresholds depend on both presentation time and mean velocity. In the latter, they depend only on presentation time.

ity are independent of mean velocity $[F(2,12)=1.79, p=$ .21 . The average threshold is about $25 \%$ change in velocity.

\section{Absolute Judgment Task}

Figure 3 shows the average setting that was judged as having a constant velocity in each condition. There was a significant bias only in the condition with a presentation time of $300 \mathrm{msec}$ and a mean velocity of $3 \mathrm{~cm} / \mathrm{sec}[t(6)=$ $-3.26, p=.02]$. This bias is negative: A decelerating dot is perceived to move at a constant velocity.

The thresholds found with the absolute judgment method (Figure 4) are very similar to the ones found with the differential judgment method (Figure 2). When expressed as accelerations, they decrease with presentation time $[F(2,12)=169.70, p<.01]$ and increase with mean velocity $[F(2,12)=158.01, p<.01]$ (Figure 4A). When expressed as percentages of change in velocity (Figure 4B), the thresholds are independent of both presentation time $[F(2,12)=1.62, p=.24]$ and mean velocity $[F(2,12)=$ $0.28, p=.76]$. The average threshold found with the absolute judgment method is about $25 \%$ change in velocity, the same as that found with the differential judgment method.

\section{DISCUSSION}

The settings in the absolute judgment task revealed a negative bias for the shortest presentation time (though significant only for the slowest targets). This is surprising, because two findings led us to expect a positive bias. First, Runeson (1974) found that targets moving at a constant speed appear to move faster at the beginning of the movement. In the present experiment, if subjects had perceived constantly moving dots as decelerating for such reasons, they would have set the dot to accelerate positively in order to see it moving at a constant speed. Second, it has been reported that negative acceleration is easier to detect than positive acceleration, at least if the stimulus moves horizontally (Babler \& Dannemiller, 1993; Calderone \& Kaiser, 1989; Gottsdankeret al., 1961; Schmerler, 1976). Such an asymmetry between the detection of positive and negative acceleration, whereby subjects are better at avoiding the latter, could lead to a positive bias in the setting. 


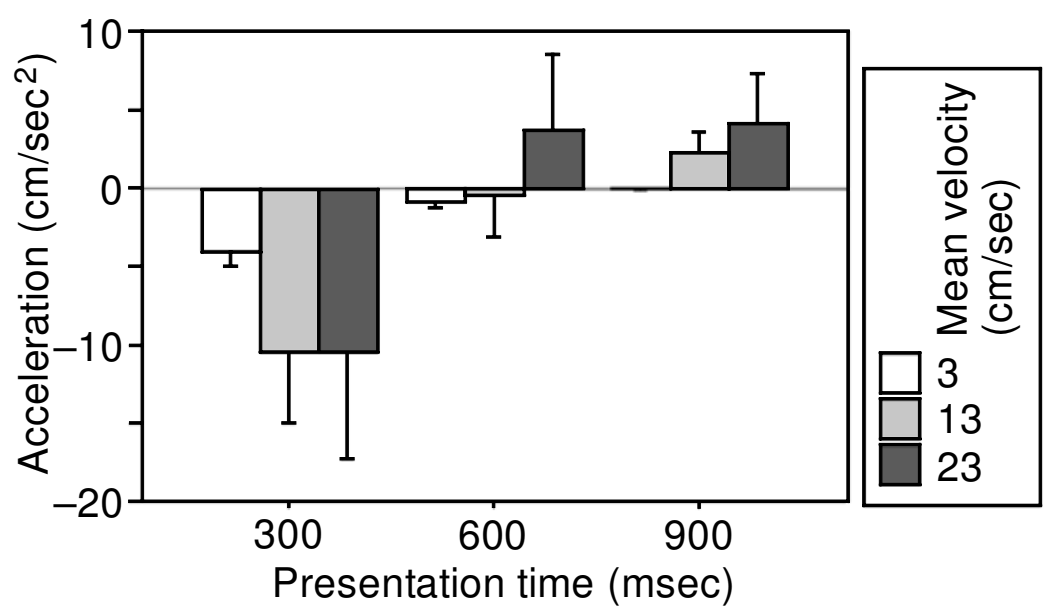

Figure 3. Average settings for each condition in the absolute judgment task. Error bars represent between-subjects standard errors. Only the average setting in the 300$\mathrm{msec}, 3-\mathrm{cm} / \mathrm{sec}$ condition is significantly different from zero.

We found that most subjects could distinguish between positive and negative acceleration when targets were presented as briefly as $300 \mathrm{msec}$. In accordance with earlier results for long presentation times (Gottsdanker et al., 1961; Schmerler, 1976), we observed that thresholds expressed as acceleration values strongly depended on the presentation time and on the mean velocity of the dot when the presentation times are short. This was so for both our methods.

The results of both methods also agreed that thresholds were more constant over different mean velocities and presentation times when they were expressed as changes in velocity as opposed to when they were expressed as acceleration values. This too is consistent with the results of Schmerler (1976), Gottsdanker et al. (1961), and Calderone and Kaiser (1989) for longer stimuli presentations. Thus subjects probably perceive the difference between the positively and negatively accelerating stimuli by comparing the initial and final velocities instead of by perceiving the acceleration itself. We will therefore further evaluate acceleration thresholds in terms of the percentages of change in velocity.

The value of the average threshold is approximately $25 \%$ change in velocity, regardless of the method used. We had not expected the values to be so similar, because both the tasks and the definition of the thresholds differed. In the differential judgment task, subjects compared positively and negatively accelerating dots, whereas in the absolute judgment task, they had to detect whether there was any acceleration present, and if so, to adjust the dot's motion. The threshold found in the differential judgment task is the acceleration at which subjects answer correctly in $75 \%$ of the cases when they have to distinguish positive from negative acceleration. The threshold found in the absolute judgment task is the standard deviation of the acceleration settings when subjects try to make the dot move at a constant velocity. Both values probably underestimate human perceptual thresholds. In the differential judgment task, we cannot tell whether subjects compared the initial and final velocities of a single dot, or whether they compared the final or initial velocities of the two dots. The compared velocities were equal in the two cases. However, comparing velocities between two dots might be easier, because a change in velocity is easier to detect if there is an interval between the presentations of the two velocities (Monen \& Brenner, 1994). In the absolute judgment task, subjects saw the dots several times, which may have reduced the thresholds.

The average $75 \%$ correct threshold found by Babler and Dannemiller (1993) was about 20\% change in velocity, which comes close to our average acceleration threshold of $25 \%$. Theirs is the best reported performance. As has been described in the introduction, other thresholds found in the literature (Calderone \& Kaiser, 1989; Gottsdanker et al., 1961; Schmerler, 1976) are substantially higher than the ones that Babler and Dannemiller and we found. In general, the value of the measured acceleration detection threshold will always depend on the specific experimental conditions. Babler and Dannemiller attributed the difference between their threshold and the ones found by others to experimental differences, one of which applies to our experiment as well. In the experiment of Babler and Dannemiller, and in our differential judgment task, subjects had to classify stimuli as positively accelerating or decelerating, without the option of them moving at a constant velocity (which was an option in the other experiments).

We already mentioned additional possible causes for our thresholds being low. We designed our stimuli in such a way that the perception of acceleration would be relatively easy (see Method). We excluded the 3 subjects with the highest thresholds (because their thresholds were beyond the reach of what could be measured for the shortest presentation times). In the differential judgment task, sub- 

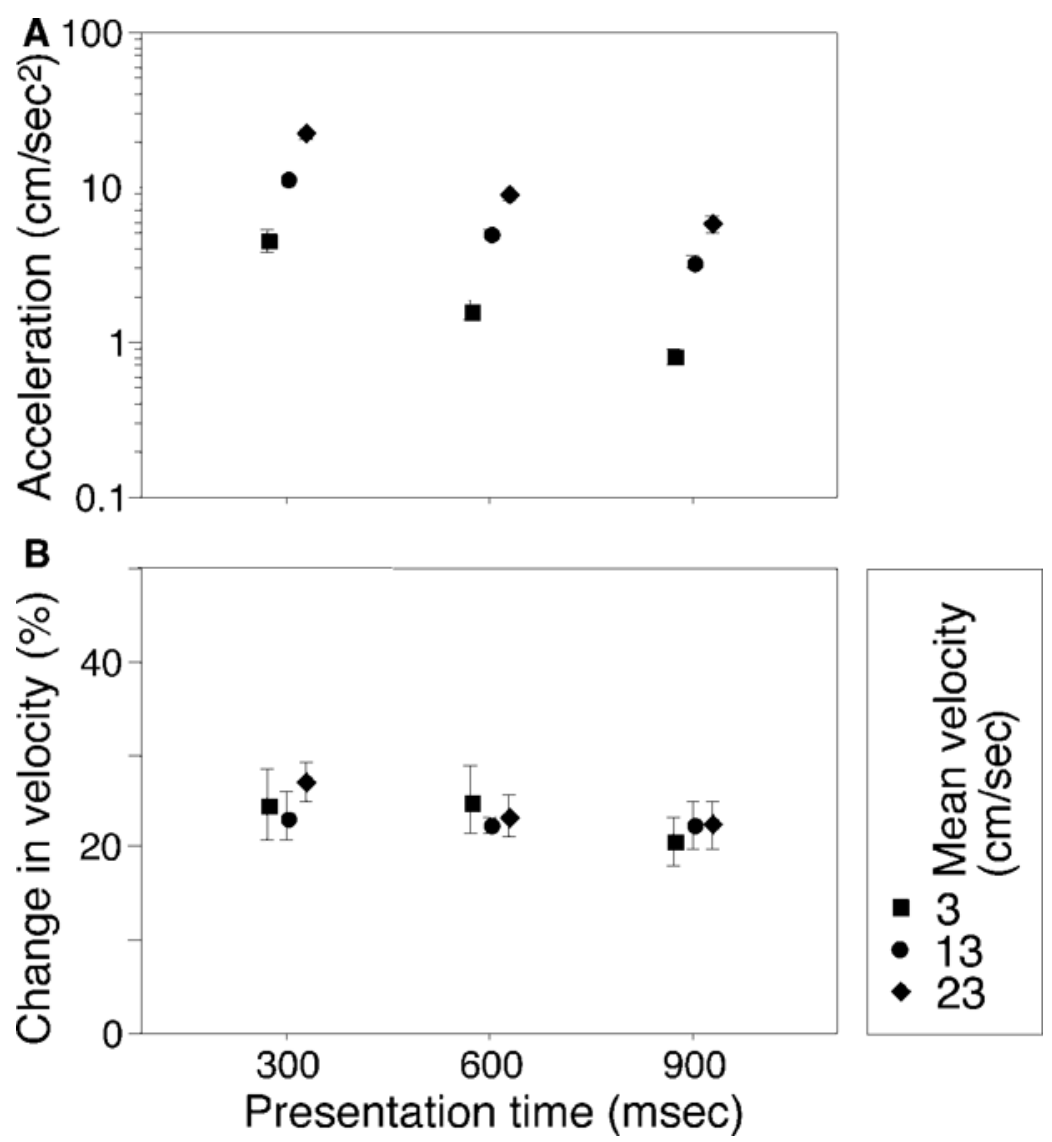

\begin{abstract}
Figure 4. Results of the absolute judgment task. Mean thresholds of 7 subjects with between-subjects standard errors for each condition. The thresholds are expressed both as rate of change in velocity $(A)$ and as percentage of change in velocity $(B)$. Thresholds depend on presentation time and mean velocity when expressed as rate of velocity change. Expressed as percentage of change in velocity, the thresholds are independent of both presentation time and mean velocity.
\end{abstract}

jects could have compared final or initial velocities between dots. In the absolute judgment task, subjects could see the stimuli as often as they liked. A final reason for our thresholds' being relatively low is the way we defined the initial and final velocity to calculate the percentage of change in velocity. Of course, the physical final and initial velocities are present only for an infinitely short time, whereas their measurement by our visual system takes time. We arbitrarily took the velocities when the dot was half visible as the initial and final velocity. In other studies, the instantaneous initial and final velocities were taken to compute the percentage of change in velocity, which results in slightly higher thresholds.

\section{Use of Acceleration for Interception}

We confirmed, for short presentation times, that the amount of change in velocity is critical for detecting acceleration, irrespective of how long it takes for that change to occur (i.e., irrespective of the acceleration). It is therefore unlikely that judgments of the acceleration itself are involved in intercepting accelerating objects moving across a frontal plane. Still, subjects could take acceleration into account in a less precise way-for example, by moving faster or reacting earlier to positively accelerating objects. In the study by Port et al. (1997) mentioned in the introduction, subjects arrived at the interception zone too early when targets decelerated and too late when they accelerated positively. Port et al. concluded that information about acceleration was not used. They claim that the accelerations that they used were above the detection threshold found with long presented stimuli. In the present study, we did not find a higher threshold for shortly presented stimuli, so one could say that the accelerations that Port et al. presented were high enough to be detected. However, in claiming that the accelerations that they used were above threshold, Port et al. considered the change in velocity between the stimulus' initial and final velocity (in the interception zone). They should have considered the change in velocity between the beginning of the target motion and the time that the movement was planned (or last adjusted). Because we do not know this time, it is impossible to determine this change in velocity. To get an in- 
dication, we computed the changes in velocity for different stimuli at the reaction time. We did so for the subject who (on the average) reacted earliest. At the reaction time, the change in velocity of all different kinds of positively accelerating stimuli was well above $25 \%$. Of the decelerating stimuli, only two out of six stimuli exceeded the threshold at this time, but the other four remained visible for a long time after the reaction time. The other subjects reacted later, so the change in target velocity was larger by the time at which they reacted. We thus confirm the conclusion of Port et al. that acceleration is not used in this kind of interception task.

It has been proposed that acceleration is used in a very simple way for catching balls (Babler \& Dannemiller, 1993; Chapman, 1968; Michaels \& Oudejans, 1992). As is depicted in Figure 5, the projection of a ball on a parabolic path accelerates negatively if the ball will land in front of the observation point and positively if it will land behind it. In principle, all that one has to do to start moving in the right direction is to move forward if the projection decelerates and backward if it accelerates positively. This is an elegant, simple strategy in which the catcher has only to distinguish between positive and negative acceleration.

Todd (1981) and Babler and Dannemiller (1993) both investigated the validity of this hypothesis, but they reached opposite conclusions. Todd presented his subjects with a simulation of the initial part of a ball approaching on a parabolic path. The object did not change size but remained very small throughout the whole trajectory. The subjects had to indicate whether the object would land in front of or on the observer. Because subjects performed poorly, Todd concluded that observers were unable to take advantage of the information contained in the acceleration. Babler and Dannemiller argued that this result might be due to the fact that the acceleration threshold of $20 \%$ change in velocity (that was found by Babler and Dannemiller themselves) was reached in only $40 \%$ of the trials. They essentially replicated Todd's experiment, taking care that their threshold was reached by the time when the target disappeared. The virtual target could also land behind the observer (which was an additional choice alternative as well). The subjects correctly indicated the landing position in about $80 \%$ of the trials. Babler and Dannemiller concluded that subjects could use the image acceleration cue effectively.

A disadvantage of these studies is that simulations were used in which only the image acceleration cue was present. It is not known how the absence of other cues that are normally present might have influenced the results. In these simulations, subjects might use the acceleration cue even though they would not use it in natural situations, where there might be other cues that (when combined) would provide the necessary information. One could think of changing image size, binocular information, image velocity, and knowledge of the ball's size. Moreover, in the judgment tasks used by Todd (1981) and Babler and Dannemiller (1993), subjects viewed the stimulus for 0.75 to $1.25 \mathrm{sec}$ and responded afterwards, without any time pressure. This is substantially different from really catching a ball, in which a quick detection and response are necessary.

Another approach to investigating whether subjects use acceleration to determine which direction they should

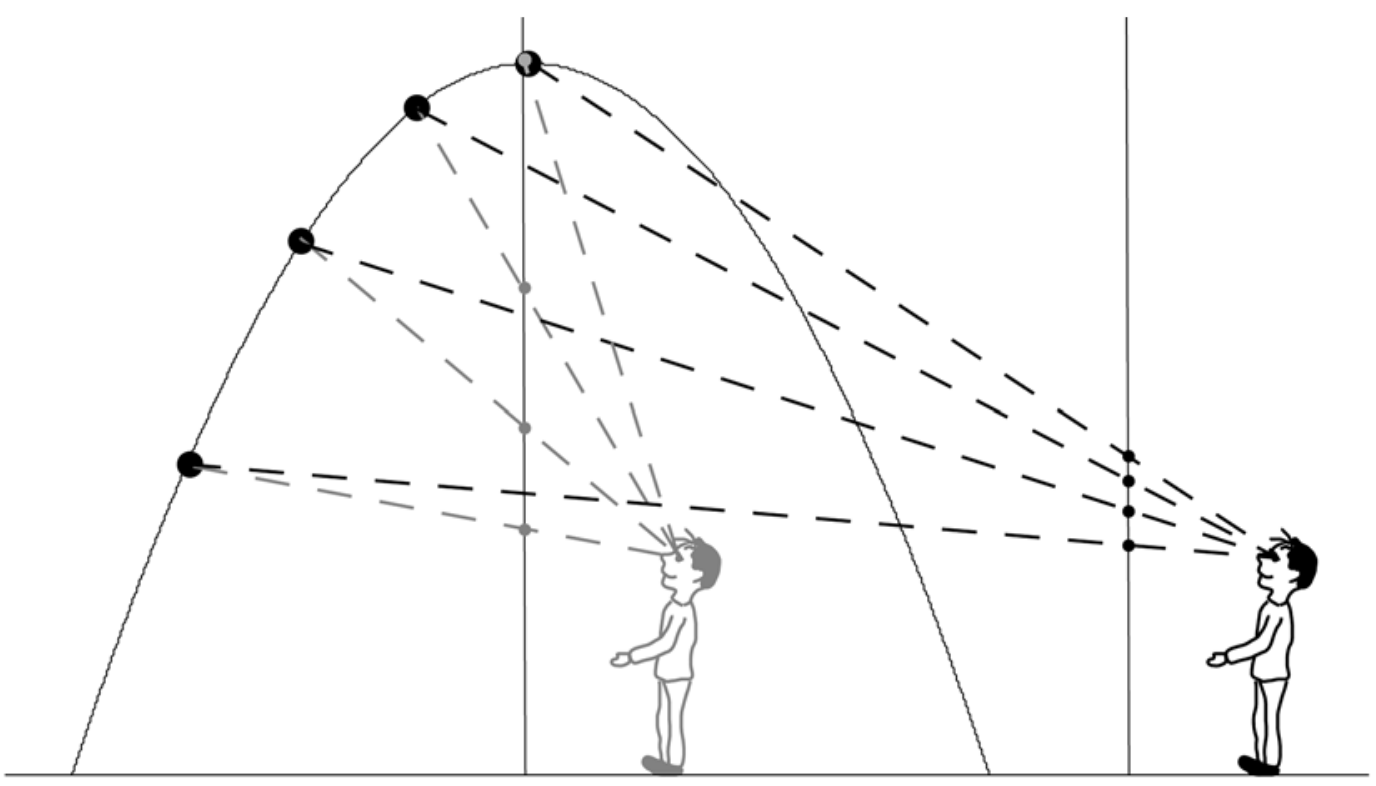

Figure 5. Schematic illustration of the acceleration cue for catching fly balls. Four positions of a ball following a parabolic path are indicated at equidistant time intervals. If the ball is destined to land in front of the observation point, its projection decelerates in the vertical direction (depicted as black dots on the vertical line). If the ball will land behind the initial observation point, the projection accelerates positively (gray dots on the vertical line). 
move in is to look at the behavior of real (competent) ball catchers. If they use the acceleration detection strategy described above, the response latencies should never be shorter than the time that it takes for the ball to reach the detection threshold for acceleration. This will now be examined.

We used raw data from a study by Oudejans, Michaels, and Bakker (1997) to see whether subjects started running only after the threshold for acceleration detection was reached. Oudejans et al. asked 12 subjects -6 experts (experienced baseball outfielders) and 6 nonexperts-to catch tennis balls. The balls were fired by a machine from behind an opaque screen. The distance between the machine and the subjects' starting position was always $18 \mathrm{~m}$. The balls followed near parabolic trajectories and were fired in the subject's sagittal plane. Half of the balls were fired to varying locations between the subject and $12 \mathrm{~m}$ in front of him and the other half were fired to locations between the subject and $11 \mathrm{~m}$ behind him, in random order. From videotapes, Oudejans et al. determined the time during which the ball was in flight, the distance that it traveled, and the direction and reaction time of the catcher's foot and head movements. The movement of one of the feet was the first detectable body movement. However, since a foot movement in a certain direction did not mean that the overall movement would be in the same direction, Oudejans et al. used the direction of initial head movement as the initial direction of locomotion. They reported that in almost half of the trials, the nonexperts started to move forward even when the balls were projected behind them. In the group of experts, this occurred in only one out of 180 trials. False starts in the other direction occurred in $6 \%$ of the trials in the expert group and $4 \%$ in the nonexpert group. The reaction time of the nonexperts was lower than that of the experts. Oudejans et al. inferred that on many occasions, the nonexperts did not respond to information about the ball's destination (they moved too early), whereas the experts did. Therefore, we examined only the trials of the experts in more detail.

To compute the moment when the velocity in the vertical direction changed by $25 \%$ for each throw, we first transformed the initial velocity of the ball into an initial optical velocity (see Michaels \& Oudejans, 1992). To arrive at the optical velocity that represents $25 \%$ change of the average velocity, we multiplied the initial optical velocity by 1.29 if the ball landed behind the starting position of the subject and 0.78 if the ball landed in front. Finally, we computed when this optical velocity was reached.

For each trial, we compared the time at which the percentage of change in velocity in the vertical direction reached $25 \%$ with the time at which subjects started to move a foot (left of Figure 6). In by far the most of the trials, the subjects reacted before threshold was reachedthat is, before they could have detected whether the ball accelerated positively or negatively. Moreover, there was no correlation between the reaction time and the time that it took for the threshold to be reached $\left(R^{2}=.01\right)$. However, since Oudejans et al. (1997) report that "a foot movement in a certain direction does not automatically mean

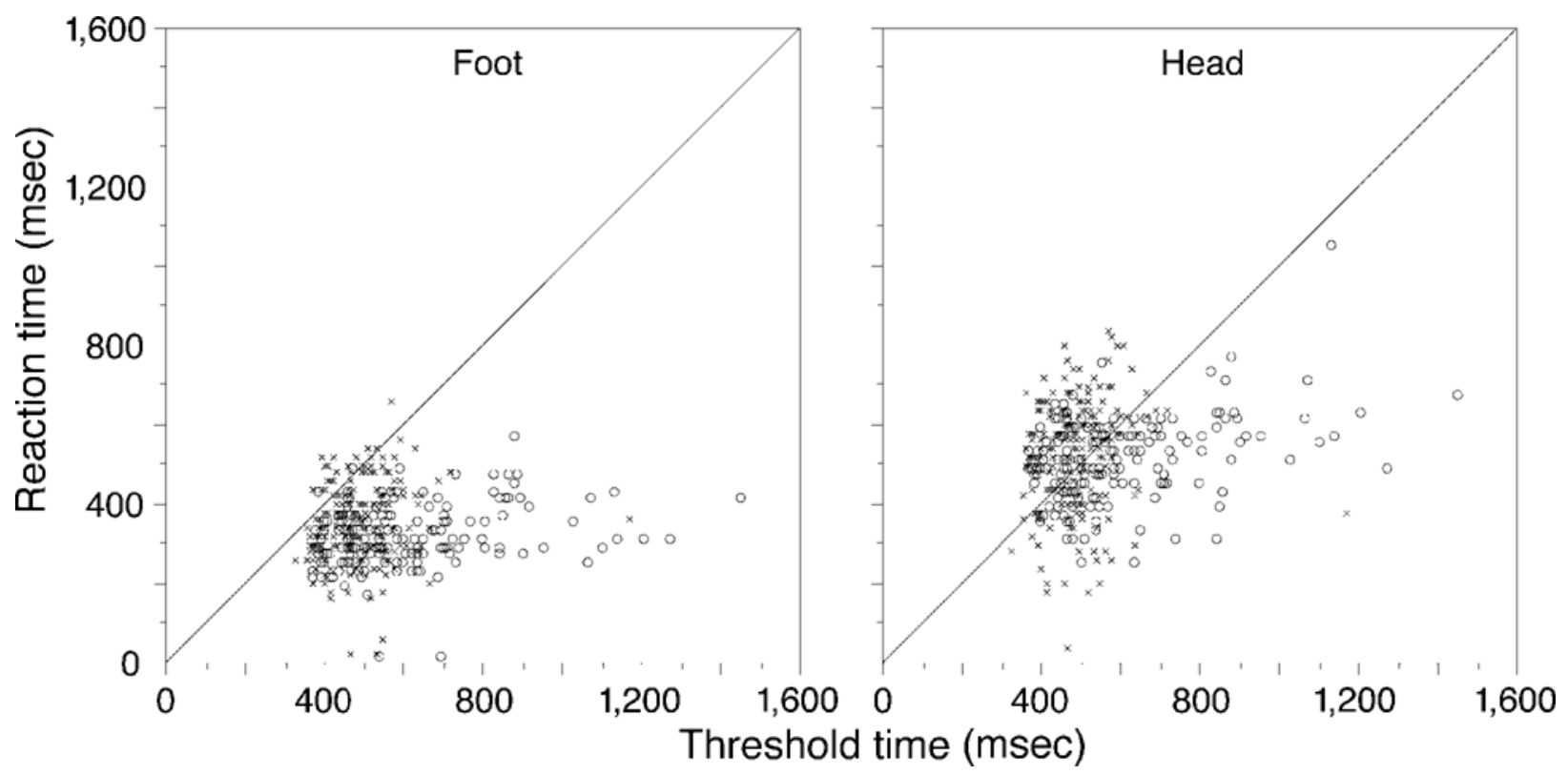

Figure 6. Reaction time of ball catchers in the study of Oudejans, Michaels, and Bakker (1997) for the foot (left) and for the head (right), plotted against the time that it took the image of the ball to reach the threshold of $25 \%$ change in velocity. Trials in which the ball landed behind the starting position of the subject are represented by circles. Trials in which the ball landed in front of the starting position are represented by crosses. Most data points are below the unity line when foot reaction time is compared with the time of threshold; with head reaction time this is about half of the points. This means that in most or half of the trials, subjects reacted before the threshold was reached, indicating that their action was not based on the detection of acceleration. 
that locomotion will be in the same direction," the subjects may not have decided for the running direction by the time they moved a foot. Therefore, we also compared the reaction times of the head with the times that it took for the acceleration detection threshold to be reached (right of Figure 6). Still, in half of the trials the reaction time was shorter than the time at which the velocity reached the detection threshold, and again there was no correlation between the reaction time and the time that it took to reach threshold $\left(R^{2}=.04\right)$. Note that if the vertical optic acceleration were used to catch balls, we would expect the reaction time in a particular trial to be in the order of $100 \mathrm{msec}$ longer than the time it would take for the threshold to be reached in that trial, because it also takes time for neural signals to result in a detectable movement.

To support the hypothesis that image acceleration is used, the acceleration detection threshold would have to have been much lower than the one that we found. However, our threshold is already an underestimate, owing to factors mentioned previously. Also, our threshold from the differential judgment task is based on a performance level of $75 \%$ correct, whereas $97 \%$ of the experts' responses were in the correct direction.

In catching balls, the motion of the image of the ball is in the vertical direction. We do not think that our threshold would have been different if we had used vertically instead of horizontally moving stimuli. Babler and Dannemiller (1993), who found a threshold about equal to ours, used vertically moving stimuli. Though Calderone and Kaiser (1989) found that an interaction between direction of motion (horizontal or vertical) and sign of acceleration affected the thresholds, the overall thresholds hardly differed between horizontally and vertically moving stimuli (if anything, the threshold for horizontally moving stimuli was slightly lower).

Thus, because subjects who are catching balls very often react before even an underestimation of the acceleration threshold is reached, we conclude that image acceleration is not used in determining in which direction to run.

\section{REFERENCES}

Babler, T. G., \& Dannemiller, J. L. (1993). Role of image acceleration in judging landing location of free-falling projectiles. Journal of Experimental Psychology: Human Perception \& Performance, 19, 15-31.
BAIRsTow, P. J. (1987). Analysis of hand movement to moving targets. Human Movement Science, 6, 205-231.

Brouwer, A., Brenner, E., \& Smeets, J. B. J. (2000). Hitting moving objects: The dependency of hand velocity on the speed of the target. Experimental Brain Research, 133, 242-248.

Calderone, J. B., \& Kaiser, M. K. (1989). Visual acceleration detection: Effect of sign and motion orientation. Perception \& Psychophysics, 45, 391-394.

Chapman, S. (1968). Catching a baseball. American Journal of Physics, 36, 868-870.

GotTSDANKer, R, Frick, J. W., \& LockARd, R. B. (1961). Identifying the acceleration of visual targets. British Journal of Psychology, 52, 31-42.

McBeath, M. K., Shaffer, D. M., \& Kaiser, M. K. (1995). How baseball outfielders determine where to run to catch fly balls. Science, $\mathbf{2 6 8}$, 569-573.

Michaels, C. F., \& Oudejans, R. R. D. (1992). The optics and actions of catching fly balls: Zeroing out optical acceleration. Ecological Psychology, 4, 199-222.

Monen, J., \& Brenner, E. (1994). Detecting changes in one's own velocity from the optic flow. Perception, 23, 681-690.

Oudejans, R. R. D., Michaels, C. F., \& Bakker, F. C. (1997). The effects of baseball experience on movement initiation in catching fly balls. Journal of Sports Sciences, 15, 587-595.

Port, N. L., Lee, D., Dassonville, P., \& Georgopoulos, A. P. (1997). Manual interception of moving targets: I. Performance and movement initiation. Experimental Brain Research, 116, 406-420.

Rosenbaum, D. A. (1975). Perception and extrapolation of speed and acceleration. Journal of Experimental Psychology: Human Perception \& Performance, 1, 395-403.

Runeson, S. (1974). Constant velocity: Not perceived as such. Psychological Research, 37, 3-23.

Schmerler, J. (1976). The visual perception of accelerated motion.Perception, 5, 167-185.

Smeets, J. B. J., \& Brenner, E. (1994). The difference between the perception of absolute and relative motion: A reaction time study. Vision Research, 34, 191-195.

ToDD, J. (1981). Visual information about moving objects. Journal of Experimental Psychology: Human Perception \& Performance, 7, 795810.

van DonkelaAR,P., Lee, R G., \& Gellman, R S. (1992). Control strategies in directing the hand to moving targets. Experimental Brain Research, 91, 151-161.

\section{NOTE}

1. We will use the term acceleration to refer to both acceleration and deceleration. To distinguish between the two, we will use positive acceleration when the speed increases and negative acceleration (or deceleration) when it decreases.

(Manuscript received May 9, 2001 revision accepted for publication February 26, 2002.) 